\title{
Adaptive Iterative Detection for the Phase-Uncertain Channel: Limited-Tree-Search Versus Truncated-Memory Detection
}

\author{
Gianluigi Ferrari, Member, IEEE, Achilleas Anastasopoulos, Member, IEEE, \\ Giulio Colavolpe, Associate Member, IEEE, and Riccardo Raheli, Member, IEEE
}

\begin{abstract}
In this paper, we consider iterative detection over bandpass channels that introduce an unknown phase rotation in the transmitted signal. This work focuses on the comparison between two adaptive detection strategies for trellis-based coded modulation: limited-tree-search (LTS) detection, obtained by reducing a tree search to a limited trellis search, and truncated-memory (TM) detection, based on channel-memory truncation, which automatically leads to a trellis search. Both strategies are used to derive trellis-based forward-backward (FB) algorithms. A quantitative analysis based on simulations, with various coding and modulation schemes, is carried out to evaluate and compare the two approaches. The results show that the channel-phase dynamics should significantly influence the choice of the detection strategy: For low-phase variations, LTS algorithms are a simple and reasonable choice, while for moderate to fast phase variations, TM algorithms show a considerable robustness.
\end{abstract}

Index Terms-Adaptive iterative detection, noncoherent detection, truncated-memory detection.

\section{INTRODUCTION}

V ARIOUS modern communication systems employ (or will employ in the future) powerful coding techniques, which consist of either serial or parallel concatenated convolutional codes (SCCCs and PCCCs) through interleavers, collectively known as turbo codes. The decoding of turbo codes is performed using iterative techniques, which offer excellent performance with limited complexity [1]-[3]. In many practical bandpass communication systems, the carrier phase needs to be estimated before the data can be decoded. This task becomes particularly difficult for turbo codes, since the operating signal-to-noise ratio (SNR) is very low. In addition, the task of phase estimation is more critical for dense constellations, as the ones typical of bandwidth efficient trellis-coded modulation (TCM) [4].

A low-complexity separate detection and decoding strategy can be realized by augmenting the coherent iterative decoder by

Manuscript received March 19, 2003; revised August 11, 2003 and December 3,2003 . This paper was presented in part at the IEEE SoftCOM2001 Workshop on Channel Coding Techniques, Ancona, Italy, October 2001, and at the IEEE Global Telecommunications Conference (GLOBECOM'02), Taipei, Taiwan, in November 2002.

G. Ferrari, G. Colavolpe, and R. Raheli are with the Department of Information Engineering, University of Parma, Parma 43100, Italy (e-mail: gianluigi.ferrari@unipr.it; giulio.colavolpe@unipr.it; riccardo.raheli@unipr.it).

A. Anastasopoulos is with the Electrical Engineering and Computer Science Department, University of Michigan, Ann Arbor, MI 48109-2122 USA (e-mail anastas@umich.edu).

Digital Object Identifier 10.1109/TVT.2004.823532 a phase estimator, e.g., a decision-directed phase-locked loop (DD-PLL) that operates on the raw coded symbols and provides a "derotated" observation to the rest of the decoder [5]. It was observed in [6] that this technique is insufficient at mediumphase dynamics. A more advanced structure consists of the receiver described above, where the phase estimator is aided by hard- or soft-decision feedback from the iterative decoder [7]. In particular, in [6], the coherent iterative decoder was augmented by a block, called the adaptive soft demodulator (A-SODEM), which processes soft decisions from the decoder and feeds back soft decisions to the decoder, while jointly recovering the phase. Thus, the A-SODEM does not explicitly account for the structure of the output sequence due to the channel code, but only implicitly, by utilizing the soft decisions generated in the iterative decoding process. Since the decoding and detection/estimation tasks are still segregated in this structure, performance degrades for high phase dynamics [6]. A similar separate detection and decoding scheme was also considered in [8], where differential encoding - rather than pilot symbol-assisted modulation - was used to transmit the coded symbols and was taken into account in the A-SODEM.

More powerful algorithms are based on combined detection and decoding strategies, where, as the term suggests, the tasks of decoding and detection/estimation are performed in a joint manner. ${ }^{1}$ One approach is to appropriately quantize the unknown phase and to apply well-known data-detection techniques (e.g., the BCJR algorithm [9]) on the supertrellis composed of the unknown data and the unknown quantized phase [10], [11]. In this paper, we do not consider this approach. Instead, we focus on combined detection and decoding strategies that treat the unknown phase as a continuous parameter. In this context, two classes of algorithms that have appeared in the literature [6], [8] will be discussed and compared. They both are forward-backward (FB) algorithms, i.e., they both consist of forward and backward add-compare-select (or sum-product) steps operating on a trellis and are aided by some sort of (implicit or explicit) per-survivor parameter estimation. The basic difference between these two groups of algorithms resides in the approach entailed by the specific detection strategy.

\footnotetext{
${ }^{1}$ It is noted that combined detection and decoding strategies do not allow the use of a channel interleaver, i.e., an interleaver following the modulator Channel interleaving is common when transmitting over a fading channel. However, since this paper considers only phase variations, channel interleaving is not necessary.
} 


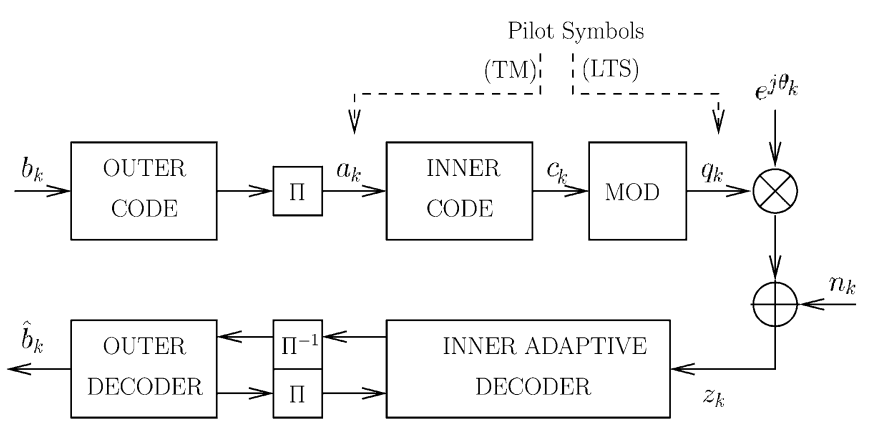

Fig. 1. Serially concatenated coding scheme with combined detection and decoding: transmitter, channel, and adaptive iterative decoder.

The first class of algorithms is related to the general approach to adaptive soft-input-soft-output (SISO) algorithms, proposed in [12] and specialized in [6], to the case of a phase-uncertain channel. The basic idea is that, since the channel memory is infinite, the generation of any soft output would require the introduction of a sequence tree, where each path leading to a different leaf would represent a specific information sequence. In [6], only a portion of the entire sequence tree is considered. In particular, the entire tree is folded/pruned to a trellis of arbitrary size (depending on the required complexity). We refer to this as the limited-tree-search (LTS) approach . It is noted that along each of the retained paths, the LTS algorithm keeps track of the entire infinite memory of the channel by means of recursive estimators, based on DD-PLLs.

The second class of algorithms is related to the work in [8], [13], and [14], where the infinite memory of the channel is " $a$ priori" truncated. Once the memory truncation is performed, the system given by the channel and a possible channel encoder collapses to a finite state machine (FSM) and an FB algorithm can be derived accordingly. As it turns out, this type of algorithm can be interpreted as performing implicit parameter estimation by means of calculating branch metrics based on a "sliding window" of observations. These algorithms will be referred to as truncated-memory (TM) algorithms. It is important to observe that since both LTS algorithms and TM algorithms are trellis-based algorithms, the main difference lies in the branch metrics.

In this paper, we propose a direct comparison between the LTS and TM detection strategies, both for combined detection and decoding and separate detection and decoding schemes-the major focus is on the structure of the algorithms, rather than on the receiver scheme (either separate or combined). The main goal of this paper is to quantify the performance difference between the two considered detection strategies. This is an interesting and relatively not clear comparison, which has seldom been treated explicitely in the literature. In fact, while a single possible detection strategy is considered and evaluated in most of the published works, in this paper we directly compare the two proposed detection strategies with the same communication schemes. Moreover, since the two detection strategies are described with similar formulations, the obtained performance comparison is even more immediate and meaningful. This allows us to gain further insights into the design of adaptive receivers for phase-uncertain channels,



Fig. 2. Parallel concatenated coding scheme with combined detection and decoding: transmitter, channel, and iterative decoder constituted by two adaptive component decoders.

making clear its dependence on the channel dynamics. In order to achieve high performance in very fast-varying channels, pilot symbols are periodically inserted in the transmitted sequence. The pilot symbols can be inserted in several points of the encoder structure. Their insertion leads to schemes that are antirotational invariant [15] and, consequently, noncoherently noncatastrophic [16]; that is, the unknown phase rotation introduced by the channel does not lead to any catastrophic behavior.

The rest of the paper is organized as follows. In Section II, the transmission systems and the channel model are described. Section III presents a summary of the LTS and TM detection strategies for the phase-uncertain channel and their specific implementation details. Numerical results are shown in Section IV and concluding remarks are drawn in Section V.

\section{SySTEMS AND CHANNEL MODEL}

The proposed schemes are presented according to the classification introduced in Section I, i.e., combined detection and decoding schemes and separate detection and decoding schemes. In the first case, we consider SCCC- and PCCC-based structures. In the second case, for the sake of conciseness, we consider a PCCC-based structure only. The proposed analysis, however, can be generalized to any code network [3].

\section{A. Combined Detection and Decoding}

The equivalent baseband discrete-time transmission system when considering an SCCC is shown in Fig. 1. The bit sequence $\left\{b_{k}\right\}$ is encoded using an outer code and is interleaved using a symbol- or bit-wise interleaver. The resulting sequence of $M$-ary symbols $\left\{a_{k}\right\}$ is coded by an inner code, producing the coded sequence $\left\{c_{k}\right\}$. The resulting coded symbols are further mapped to the complex symbols $\left\{q_{k}\right\}$ and are transmitted 
over an additive white Gaussian noise (AWGN) channel that, in addition, introduces an unknown carrier phase offset. The statistics of the phase process need not be specified at this point. The complex equivalent signal can be written, after a suitable discretization process, as

$$
z_{k}=q_{k} e^{j \theta_{k}}+n_{k}
$$

where $\left\{\theta_{k}\right\}$ represents the discrete-time phase process ${ }^{2}$ and $\left\{n_{k}\right\}$ are independent and identically distributed complex noise samples with independent real and imaginary components of equal variance $N_{0} / 2$. The receiver consists of an adaptive inner block that jointly estimates the phase and produces soft information on symbols $\left\{a_{k}\right\}$ and a nonadaptive outer block that produces soft decisions on $\left\{a_{k}\right\}$, as well as hard decisions for $\left\{b_{k}\right\}$. The details of the inner adaptive decoder, which can be either an LTS or a TM algorithm, are discussed in the next section.

In Fig. 2, a transmission scheme employing a PCCC is shown. In this case, the PCCC is constituted by two component recursive systematic convolutional (RSC) codes. For simplicity, rate-1/2 RSC codes are considered. After possible puncturing, the sequences of information and coded bits are serialized, mapped to a binary phase-shift keying (BPSK) constellation and transmitted over the channel. At the receiver side, the turbo decoder consists of two adaptive component decoders. It should be emphasized at this point that a combined detection scheme for PCCCs, such as the one described here, can become quite complicated when higher order constellations are used, as was demonstrated in [6]. Thus, only BPSK modulation is considered in this case, while higher modulations are considered in conjunction with separate detection and decoding, as described in the following subsection.

\section{B. Separate Detection and Decoding}

Separate detection and decoding schemes can be constructed by simply augmenting the coherent decoder with an A-SODEM module. Such a scheme, considered in Fig. 3, is desirable for PCCCs combined with high-order constellations. In particular, in this figure we refer to a binary turbo code with rate $1 / 3$ (without puncturing) or $1 / 2$ (with puncturing). At each epoch, three or two output bits are mapped to an 8-ary phase-shift keying (8-PSK) or a quaternary phase-shift keying (QPSK) symbol $q_{k}$, respectively. At the receiver side, there is an inner A-SODEM that recovers the unknown phase rotation and generates reliability values on each component bit of a modulated symbol, relying on the transmitted pilot symbols. These soft values are passed to a turbo decoder.

\section{Detection STRATEGIES FOR THE PHASE-UNCERTAIN CHANNEL}

Upon the assumption of perfect channel-state information (CSI) at the receiver, the channel-phase realization is known. In this case, corresponding to coherent detection, the branch

${ }^{2}$ It is assumed that the continuous-time phase process is slow as compared with the symbol duration and, thus, a symbol-spaced matched filter provides sufficient statistics for detection.

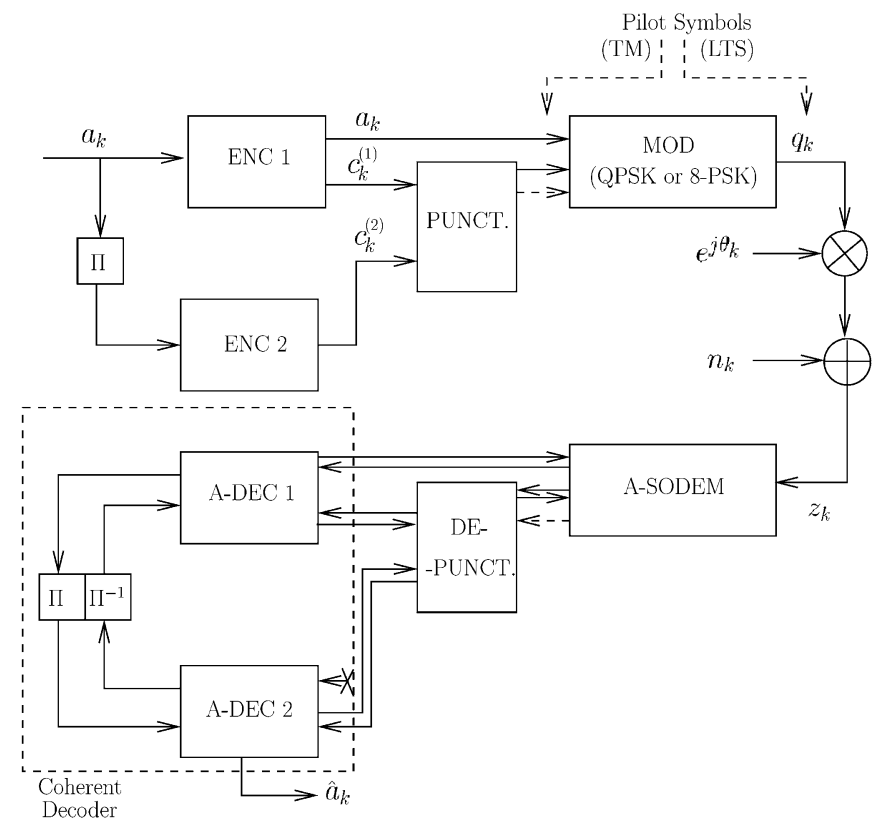

Fig. 3. Parallel concatenated coding scheme with separate detection and decoding: transmitter, channel, and iterative decoder constituted by the concatenation of an A-SODEM and a coherent turbo decoder.

metrics of an FB algorithm can be computed exactly, based on the known Gaussian distribution of the additive noise. If CSI is not available at the receiver, the detection problem becomes more complicated. In this case, however, the concept of per-survivor processing (PSP), which is a general approach to detection where the unknown parameters are estimated along each path of the trellis of a suitable FSM [17], can be applied to derive FB algorithms.

In this section, we briefly summarize two options for generating symbol-by-symbol soft decision information in the presence of an unknown channel phase, based on the works in [6] and [14]. Our goal is to clearly show how the formulations of two substantially different detection approaches can be unified, which simplifies the task of properly comparing the two detection strategies. The development is based on an isolated FSM that models different blocks, depending on the particular encoding/decoding strategy. For instance, it models

1) the inner encoder, in the case of combined detection and decoding of SCCCs;

2) each of the constituent encoders, in the case of combined detection and decoding of PCCCs;

3 ) the uncoded sequence in the case of separate detection and decoding.

This FSM can also model expanded versions of the above blocks, i.e., we might want to utilize an FSM with 16 states to describe a four-state encoder, in order to account for the memory due to the unknown phase process.

The FSM is defined by a state $s_{k}$ and a transition $t_{k}=\left(s_{k}, a_{k}\right)$. At epoch $k$, the output symbol $q_{k}$ is emitted. At the receiver, a suitable front-end processing is considered, leading to one sample $z_{k}$ per symbol interval, as shown in (1). The notation $\mathbf{z}_{k_{1}}^{k_{2}}$ is used to denote a sequence of symbols $\left(z_{k_{1}}, \ldots, z_{k_{2}}\right), k_{1} \leq k_{2}$. Finally, we assume a length $K$ information sequence and denote for brevity $\mathbf{a}_{1}^{K}=\mathbf{a}$ (similarly, 
$\left.\mathbf{x}_{1}^{K}=\mathbf{x}\right)$. The starting point of both soft-output detection strategies is the calculation of the a posteriori probability

$$
P\left\{a_{k} \mid \mathbf{z}\right\} \propto \sum_{\mathbf{a}: a_{k}} p(\mathbf{z} \mid \mathbf{a}) P\{\mathbf{a}\}
$$

where the symbol $\propto$ indicates proportionality and the notation a : $a_{k}$ indicates all information sequences compatible with $a_{k}$. The different detection strategies are based on different approaches to the computation of the conditional probability density function (pdf) $p(\mathbf{z} \mid \mathbf{a})$ on the right-hand side of (2).

\section{A. LTS Adaptive FB Algorithms}

The basic idea of the LTS approach is to view the set of all possible sequences $\mathbf{a}=\mathbf{a}_{1}^{K}$ as paths of a depth- $K$ tree. Searching over all these sequences is required to obtain the exact solution to the detection problem. The LTS algorithm approximates this solution by reducing the "optimal" tree search over all possible sequences a to a limited number of sequences, according to a tree-pruning strategy. In particular, by choosing the Viterbi algorithm as the pruning strategy, the sequence tree reduces to a trellis where the generic state is $S_{k} \triangleq\left(s_{k-N+1}, a_{k-N+1}, \ldots, a_{k-1}\right)$. The parameter $N \geq 1$ is a design choice and determines the expanded trellis size, with respect to the encoder trellis, whose state ${ }^{3}$ is $s_{k}$. A transition in the expanded trellis is indicated by $T_{k}=\left(S_{k}, a_{k}\right)=\left(s_{k-N+1}, a_{k-N+1}, \ldots, a_{k}\right)$. In this case, the quantity on the right-hand side of (2) can be approximated as [6]

$$
\begin{aligned}
P\left\{a_{k} \mid \mathbf{z}\right\} \widetilde{\propto} & \sum_{T_{k}: a_{k}} p\left[\mathbf{z}_{1}^{k-1} \mid S_{k}, \hat{\theta}_{k}^{f}\left(S_{k}\right)\right] P\left\{S_{k}\right\} \\
& \cdot p\left[z_{k} \mid T_{k}, \hat{\theta}_{k}^{f}\left(S_{k}\right), \hat{\theta}_{k+1}^{b}\left(S_{k+1}\right)\right] P\left\{a_{k}\right\} \\
& \cdot p\left[\mathbf{z}_{k+1}^{K} \mid S_{k+1}, \hat{\theta}_{k+1}^{b}\left(S_{k+1}\right)\right]
\end{aligned}
$$

where $\hat{\theta}_{k}^{i}\left(S_{k}\right)$ and $i \in\{f, b\}$ are forward- and backward-phase estimates, respectively, which are obtained as described in the following. For the sake of simplicity, we will simply use the notation $\hat{\theta}_{k}^{i}$ to indicate an estimate of $\theta_{k}$-the dependence on a state $S_{k}$ should be clear from the context. By defining

$$
\begin{aligned}
\alpha_{k}\left(S_{k}\right) & \triangleq p\left(\mathbf{z}_{1}^{k-1} \mid S_{k}, \hat{\theta}_{k}^{f}\right) P\left\{S_{k}\right\} \\
\gamma_{k}^{\mathrm{LTS}, c}\left(T_{k}, \hat{\theta}_{k}^{f}, \hat{\theta}_{k+1}^{b}, z_{k}\right) & \triangleq p\left(z_{k} \mid T_{k}, \hat{\theta}_{k}^{f}, \hat{\theta}_{k+1}^{b}\right) P\left\{a_{k}\right\} \\
\beta_{k+1}\left(S_{k+1}\right) & \triangleq p\left(\mathbf{z}_{k+1}^{K} \mid S_{k+1}, \hat{\theta}_{k+1}^{b}\right)
\end{aligned}
$$

the soft output on the right-hand side of (3), generated by an adaptive LTS algorithm that we denote by $S^{L T S}\left[a_{k}\right]$, can be written as

$$
\begin{aligned}
S^{\operatorname{LTS}}\left[a_{k}\right]= & \sum_{T_{k}: a_{k}} \alpha_{k}\left(S_{k}\right) \gamma_{k}^{\mathrm{LTS}, c}\left(T_{k}, \hat{\theta}_{k}^{f}, \hat{\theta}_{k+1}^{b}, z_{k}\right) \\
& \cdot \beta_{k+1}\left(S_{k+1}\right) .
\end{aligned}
$$

${ }^{3} N=1$ corresponds to the case $S_{k}=s_{k}$, i.e., there is no trellis expansion.
It is important to remark that we explicitly consider the dependence on the observation and the phase estimate only in the expression of the branch metric ${ }^{4} \gamma_{k}^{\mathrm{LTS}, c}$, assuming an implicit dependence on it in the expressions of the quantities $\alpha_{k}$ and $\beta_{k+1}$. In [6], it is shown that the branch metric $\gamma_{k}^{\mathrm{LTS}, c}\left(T_{k}\right)$ characterizing the completion operation (7) can be written as

$$
\gamma_{k}^{\mathrm{LTS}, c}\left(T_{k}, \hat{\theta}_{k}^{f}, \hat{\theta}_{k+1}^{b}, z_{k}\right)=\gamma_{k}^{\mathrm{LTS}}\left(T_{k}, \hat{\theta}, z_{k}\right) b\left(\hat{\theta}_{k}^{f}, \hat{\theta}_{k+1}^{b}\right)
$$

where $\hat{\theta}$ is either $\hat{\theta}_{k}^{f}$ or $\hat{\theta}_{k+1}^{b}$ (see [6] for details) and

$$
\begin{aligned}
\gamma_{k}^{\mathrm{LTS}}\left(T_{k}, \hat{\theta}, z_{k}\right) & =P\left\{a_{k}\right\} \exp \left\{\frac{2 \Re\left(z_{k} e^{-j \hat{\theta}} q_{k}^{*}\right)-\left|q_{k}\right|^{2}}{2 \sigma^{2}}\right\} \\
b\left(\hat{\theta}_{k-1}^{f}, \hat{\theta}_{k}^{b}\right) & =\exp \left(-\frac{1-\lambda}{\lambda(2-\lambda)} \mid e^{j \hat{\theta}_{k-1}^{f}-\left.e^{\hat{\theta}_{k}^{b}}\right|^{2}}\right)
\end{aligned}
$$

where the meaning of the parameter $\lambda$, which is related to the recursive phase-estimation strategy, is clarified below. The quantities $\alpha_{k}\left(S_{k}\right)$ and $\beta_{k+1}\left(S_{k+1}\right)$ in (7) can be computed by means of forward and backward recursions, during which the forward and backward phase estimates, respectively, are computed.

Let us consider the forward recursion. In general, it can be written as

$$
\alpha_{k+1}\left(S_{k+1}\right)=\sum_{T_{k}: S_{k+1}} \alpha_{k}\left(S_{k}\right) \gamma_{k}^{\mathrm{LTS}}\left(t_{k}, \hat{\theta}_{k}^{f}, z_{k}\right) .
$$

The phase estimate $\hat{\theta}_{k}^{f}$ is updated in a PSP fashion, using a firstorder PLL. The PLL update equation is given by

$$
\hat{\theta}_{k+1}^{f}=\hat{\theta}_{k}^{f}\left(S_{k}^{(\max )}\right)+\lambda \Im\left\{z_{k} q_{k}^{*} e^{-j \hat{\theta}_{k}^{f}\left(S_{k}^{(\max )}\right)}\right\}
$$

where the transition $T_{k}^{(\max )}$ (and the corresponding beginning state $S_{k}^{(\max )}$ ) is the one determined by an add-compare-select operation similar to the one in (11), derived by exchanging the summation operation $\sum_{T_{k}: S_{k+1}}$ by a maximization operation $\max _{T_{k}: S_{k+1}}$. Finally, the parameter $\lambda$ determines the PLL bandwidth. The backward recursion, during which a backward phase estimate $\hat{\theta}_{k}^{b}$ is updated, is similar to the forward recursion.

At this point, we would like to emphasize that the LTS algorithm only keeps and updates a limited number of phase estimates. Each of these estimates corresponds to each of the survivors, i.e., each of the tree paths that are explored in the limited tree-search procedure. However, due to the recursive nature of the parameter update equations, the entire memory of the channel is retained in each of these estimates. [For this reason, this class of adaptive algorithms is also referred to as closed-loop (CL) adaptive algorithms.] This is a unique characteristic of the LTS algorithm and is also one of its drawbacks for fast phase dynamics, as will be evident from the results of Section IV. In all the considered transmission schemes with the LTS detection strategy, pilot symbols are inserted in the output-modulated symbols, as indicated in Figs. 1-3.

\footnotetext{
4The term "metric" usually refers to the logarithmic domain. For extension, we use the same term that is also in the probabilistic domain.
} 


\section{B. TM Adaptive FB Algorithms}

A TM-FB algorithm can be derived by approximating the expression in (2) as [14]

$$
\begin{array}{r}
P\left\{a_{k} \mid \mathbf{z}\right\} \approx \sum_{T_{k}: a_{k}} p\left(\mathbf{z}_{1}^{k-1} \mid S_{k}\right) P\left\{S_{k}\right\} p\left(z_{k} \mid T_{k}, \mathbf{z}_{k-N+1}^{k-1}\right) \\
\cdot P\left\{a_{k}\right\} p\left(\mathbf{z}_{k+1}^{K} \mid S_{k+1}, \mathbf{z}_{k-N+2}^{k}\right)
\end{array}
$$

where the expanded state and transition are defined as in the LTS case, i.e., $S_{k}=\left(s_{k-N+1}, a_{k-N+1}, \ldots, a_{k-1}\right)$, and $T_{k}=$ $\left(S_{k}, a_{k}\right)$. However, in this case, the parameter $N$ is not chosen to limit the search over a reduced number of information sequences, as in the case of the LTS algorithm. Rather, the parameter $N$ is chosen to partially account for the memory induced by the unknown channel-phase rotation through a window of $N$ symbols. In particular, the integer $N$, originally introduced as the phase-memory parameter in [18], quantifies the amount of channel memory taken into account in the trellis diagram of the considered detection block. By defining

$$
\begin{aligned}
\alpha_{k}\left(S_{k}\right) & \triangleq p\left(\mathbf{z}_{1}^{k-1} \mid S_{k}\right) P\left\{S_{k}\right\} \\
\gamma_{k}^{\mathrm{TM}}\left(T_{k}, \mathbf{z}_{k-N+1}^{k}\right) & \triangleq p\left(z_{k} \mid T_{k}, \mathbf{z}_{k-N+1}^{k-1}\right) P\left\{a_{k}\right\} \\
\beta_{k+1}\left(S_{k+1}\right) & \triangleq p\left(\mathbf{z}_{k+1}^{K} \mid S_{k+1}, \mathbf{z}_{k-N+2}^{k}\right)
\end{aligned}
$$

it follows that the soft output on the right-hand side of (3), generated by a TM algorithm, that is denoted by $S^{\mathrm{TM}}\left[a_{k}\right]$, can be written as

$$
S^{\mathrm{TM}}\left[a_{k}\right]=\sum_{T_{k}: a_{k}} \alpha_{k}\left(S_{k}\right) \gamma_{k}^{T M}\left(T_{k}, \mathbf{z}_{k-N+1}^{k}\right) \beta_{k+1}\left(S_{k+1}\right) .
$$

It is possible to show that the quantities $\alpha_{k}\left(S_{k}\right)$ and $\beta_{k+1}\left(S_{k+1}\right)$ can be calculated via forward and backward recursions, respectively, as [14]

$$
\begin{aligned}
\alpha_{k+1}\left(S_{k+1}\right) & =\sum_{T_{k}: S_{k+1}} \alpha_{k}\left(S_{k}\right) \gamma_{k}^{\mathrm{TM}}\left(T_{k}, \mathbf{z}_{k-N+1}^{k}\right) \\
\beta_{k}\left(S_{k}\right) & =\sum_{T_{k}: S_{k}} \beta_{k+1}\left(S_{k+1}\right) \gamma_{k}^{\mathrm{TM}}\left(T_{k}, \mathbf{z}_{k-N+1}^{k}\right) .
\end{aligned}
$$

At this point, we emphasize that once the memory truncation is performed in (13), the TM-FB algorithm runs on a finite trellis (defined by the states $\left\{S_{k}\right\}$ ). In other words, no additional tree pruning is required. This is a subtle (since the final formulation of the two approaches is very similar) but substantial difference from the LTS algorithm described in the previous section.

The specific expression for the branch metric $\gamma_{k}^{\mathrm{TM}}$ depends on the channel model. Following the approach in [18] and [19], the phase rotation will be modeled as a random variable with uniform distribution in $[0,2 \pi)$, constant for the window of length $N$. This leads to the following expression for the branch metric:

$$
\begin{aligned}
\gamma_{k}^{\mathrm{TM}}\left(T_{k}, \mathbf{z}_{k-N+1}^{k}\right) \propto & P\left\{a_{k}\right\} \exp \left(-\frac{\left|q_{k}\right|^{2}}{2 \sigma^{2}}\right) \\
& \cdot \frac{I_{0}\left(\frac{1}{\sigma^{2}}\left|\sum_{i=0}^{N-1} z_{k-i} q_{k-i}^{*}\right|\right)}{I_{0}\left(\frac{1}{\sigma^{2}}\left|\sum_{i=1}^{N-1} z_{k-i} q_{k-i}^{*}\right|\right)} .
\end{aligned}
$$

Even though the above expression is derived under a simplifying assumption for the phase process, the memory-truncation assumption allows the algorithm to cope with more practical time-varying phase processes, as will be demonstrated in Section IV, where the derived algorithm will be applied to channels characterized by more realistic phase process models (e.g., random walk).

Comparing the expressions in (9) and (20), it can be observed that there is no explicit phase estimate involved in the branch metric $\gamma_{k}^{\mathrm{TM}}$. However, by proper manipulations and slight approximations, it is possible to interpret this branch metric as implicitly performing phase estimation, based on a window of consecutive observations. More precisely, observing that $I_{0}(x) \approx e^{x}$ for sufficiently large $x$ and that, for a generic complex number $c,|c|=c e^{-j L c}=\Re\left(c e^{-j L c}\right)$, (20) can be approximated as

$$
\begin{aligned}
\gamma_{k}^{\mathrm{TM}} & \left(T_{k}, \mathbf{z}_{k-N+1}^{k}\right) \\
\approx & P\left\{a_{k}\right\} \exp \left\{-\frac{\left|q_{k}\right|^{2}}{2 \sigma^{2}}\right\} \\
& \cdot \exp \left(\frac{\left|\sum_{i=0}^{N-1} z_{k-i} q_{k-i}^{*}\right|}{\sigma^{2}}\right) \\
& \cdot \exp \left(-\frac{\left|\sum_{i=1}^{N-1} z_{k-i} q_{k-i}^{*}\right|}{\sigma^{2}}\right) \\
= & P\left\{a_{k}\right\} \exp \left\{-\frac{\left|q_{k}\right|^{2}}{\left.2 \sigma^{2}\right\}}\right) \\
& \cdot \exp \left(\frac{\sum_{i=0}^{N-1} \Re\left\{z_{k-i} e^{-j \hat{\theta}_{k}^{(N)}} q_{k-i}^{*}\right\}}{\sigma^{2}}\right) \\
& \cdot \exp \left(-\frac{\sum_{i=1}^{N-1} \Re\left\{z_{k-i} e^{-j \hat{\theta}_{k}^{(N-1)}} q_{k-i}^{*}\right\}}{\sigma^{2}}\right)
\end{aligned}
$$

where the implicit phase estimates are defined as

$$
\begin{aligned}
\hat{\theta}_{k}^{(N)} & =\arg \left(\sum_{i=0}^{N-1} z_{k-i} q_{k-i}^{*}\right) \\
\hat{\theta}_{k}^{(N-1)} & =\arg \left(\sum_{i=1}^{N-1} z_{k-i} q_{k-i}^{*}\right) .
\end{aligned}
$$

If $\hat{\theta}_{k}^{(N)} \approx \hat{\theta}_{k}^{(N-1)}$, then (21) can be further approximated as

$$
\begin{array}{r}
\gamma_{k}^{\mathrm{TM}}\left(T_{k}, \mathbf{z}_{k-N+1}^{k}\right) \approx P\left\{a_{k}\right\} \exp \left\{-\frac{\left|q_{k}\right|^{2}}{2 \sigma^{2}}\right\} \\
\cdot \exp \left\{\frac{\Re\left\{z_{k} e^{-j \hat{\theta}_{k}^{(N)}} q_{k}^{*}\right\}}{\sigma^{2}}\right\} .
\end{array}
$$

For a constant channel-phase rotation, the last approximation is sensible for $N$ large enough [20], [21], while for a timevarying channel-phase rotation, an optimal value of $N$ exists, as will be evident from the numerical results in Section IV. Moreover, the final expression in (24) is formally identical to the corresponding metric for the LTS approach in (9) and 


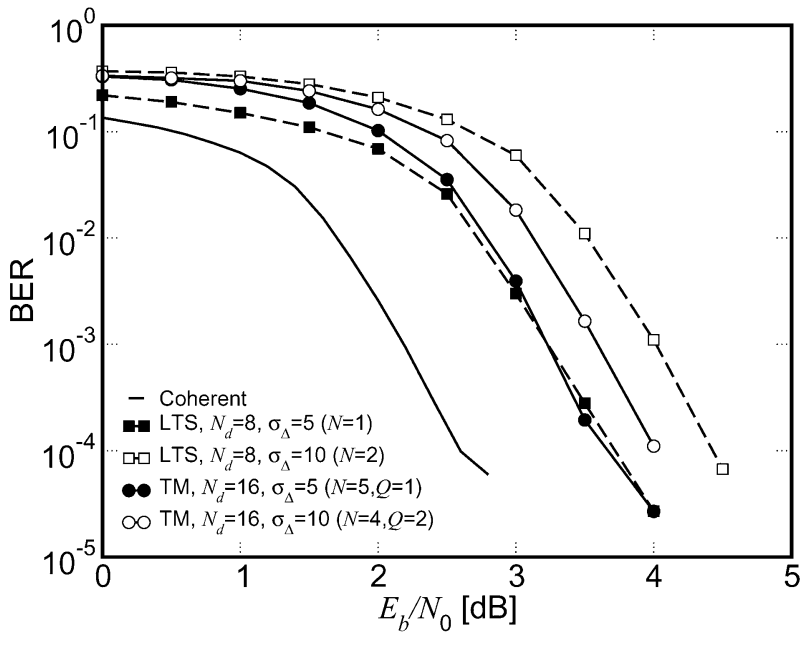

Fig. 4. BER of an SCCC with TM and LTS inner decoding algorithm, for phase-jitter standard-deviation $\sigma_{\Delta}=5^{\circ}$ and $\sigma_{\Delta}=10^{\circ}$. The spectral efficiency is $1 \mathrm{~b} / \mathrm{s} / \mathrm{Hz}$. For comparison, the performance of the equivalent coherent scheme is shown. In all cases, ten decoding iterations are considered.

clearly shows the connection between $\gamma_{k}^{\mathrm{TM}}\left(T_{k}, \mathbf{z}_{k-N+1}^{k}\right)$ and the implicit TM phase estimate $\hat{\theta}_{k}^{(N)}$ in (22). Since the TM phase estimate depends on the current observation window, two consecutive estimates $\hat{\theta}_{k-1}^{(N)}$ and $\hat{\theta}_{k}^{(N)}$ are not explicitly related by a recursive formula. In this sense, it possible to interpret the phase-estimation strategy embedded in (24) as open-loop (OL) phase estimation, as opposed to the CL estimation in LTS algorithms. In all the considered transmission schemes with the TM detection strategy, pilot symbols are inserted in the input information stream, as indicated in Figs. 1-3.

As can be seen from the definition of the receiver state $S_{k}$, one of the major drawbacks of the TM algorithm is the exponential increase of the receiver number of states as a function of the phase memory parameter $N$. In other words, both the channel memory and the trellis size are controlled by a single design parameter $N$. This drawback can be overcome by applying recently introduced state-reduction techniques for FB algorithms [14], [22], [23]. The receiver trellis state is redefined as $\Sigma_{k} \triangleq\left(s_{k-Q+1}, a_{k-Q+1}, \ldots, a_{k-1}\right)$, where the reduced-state parameter $Q<N$ quantifies the state reduction. In this way, the window size $N$ (which determines the quality of the implicit phase estimate) can be chosen independently of the trellis size (which is controlled by the parameter $Q$ and basically dictates the complexity of the algorithm).

\section{NUMERICAL RESULTS}

The performance of the proposed receivers is assessed by means of computer simulations mainly in terms of bit-error rate (BER) and frame-error rate (FER) versus $E_{b} / N_{0}, E_{b}$ being the received energy per information bit. The SNR loss due to the insertion of pilot symbols is accounted for in all the results presented herein. In all cases, $N_{p}=1$ pilot symbol is inserted every $N_{d}$ symbols. The performance of the considered systems under dynamic channel conditions is investigated. The time-varying phase process $\left\{\theta_{k}\right\}$ used in the simulations is a random walk with independent Gaussian increments, with variance over a signaling interval equal to $\sigma_{\Delta}^{2}$. In the following, we will also assume that any adaptive FB algorithm is in the min-sum form (although the algorithms were developed in the sum-product form in Section III), while any coherent FB algorithm is the standard BCJR algorithm [9]. In all presented results, we assume that the initial forward and backward phase estimates (only forward in the TM case and both in the LTS case) are ideal. This assumption is justified, since insertion of an initial and a final training sequence results in negligible bandwidth efficiency and energy loss for the considered code-word lengths. It is noted that, in all the systems examined in this work, a number of simulations were run to roughly optimize the different system parameters (e.g., $N, Q, N_{d}, \lambda$, etc.). However, for conciseness, only a small number of these results are presented in order to demonstrate the main conclusions.

The LTS and TM algorithms are first compared considering iterative decoding of two SCCCs using combined detection and decoding. The first SCCC consists of an outer four-state rate-1/2 convolutional code connected through a length-1024 pseudorandom interleaver to an inner four-state rate- $2 / 3$ convolutional code. ${ }^{5}$ The respective generator matrices are given by

$$
G_{\mathrm{o}}(D)=\left[1 \frac{1+D^{2}}{1+D+D^{2}}\right] \quad G_{\mathrm{i}}(D)=\left[\begin{array}{ccc}
1 & 0 & \frac{1+D^{2}}{1+D+D^{2}} \\
0 & 1 & \frac{1+D}{1+D+D^{2}}
\end{array}\right]
$$

The output symbols are mapped to an 8-PSK constellation with natural mapping, resulting in an overall code of spectral efficiency $1 \mathrm{~b} / \mathrm{s} / \mathrm{Hz}$. The numerical results for this system, in terms of BER, are shown in Fig. 4. For comparison, the idealized performance of the equivalent coherent receiver, i.e., with perfect phase reference, is also shown. In all cases, ten decoding iterations are considered. In the TM case, for increasing phase jitter, the performance improves by reducing the window size $N\left(N=5\right.$ for $\sigma_{\Delta}=5^{\circ}$ and $N=4$ for $\left.\sigma_{\Delta}=10^{\circ}\right)$ and the state-reduction level $\left(Q=1\right.$ for $\sigma_{\Delta}=5^{\circ}$ and $Q=2$ for $\left.\sigma_{\Delta}=10^{\circ}\right)$. For the case of LTS-based phase estimation, the considered number of decoder states is 4 and 16 , for $\sigma_{\Delta}=5^{\circ}$ and $\sigma_{\Delta}=10^{\circ}$, respectively, so that the overall complexity of the LTS- and TM-based receivers is roughly the same. Simulation results show that for $\sigma_{\Delta}=5^{\circ}$, the performance of the LTS- and TM-based receivers are almost identical at BER values of practical importance. When increasing the phase jitter to $10^{\circ}$, the LTS scheme shows a performance loss of $0.5 \mathrm{~dB}$ with respect to the TM scheme. A possible explanation for this degradation is that LTS estimation may result in losses of lock, from which it is difficult to recover. We remark that the LTS scheme requires a double number of pilot symbols to obtain a performance similar to that of the TM scheme. However, with the considered values of $N_{d}$ in the two cases (16 for the TM scheme and eight for the LTS scheme), in order to support the same information rate, the LTS scheme requires a bandwidth expansion of only $7 \%$ with respect to the TM scheme. Similar conclusions can be drawn by looking at the FER curves (not shown here).

The second considered SCCC consists of an outer four-state rate-2/3 nonrecursive convolutional code and an inner four-state rate-3/3 recursive convolutional code, connected through a

\footnotetext{
${ }^{5}$ The constituent codes in all SCCC and PCCC schemes examined herein are properly terminated using tail bits.
} 


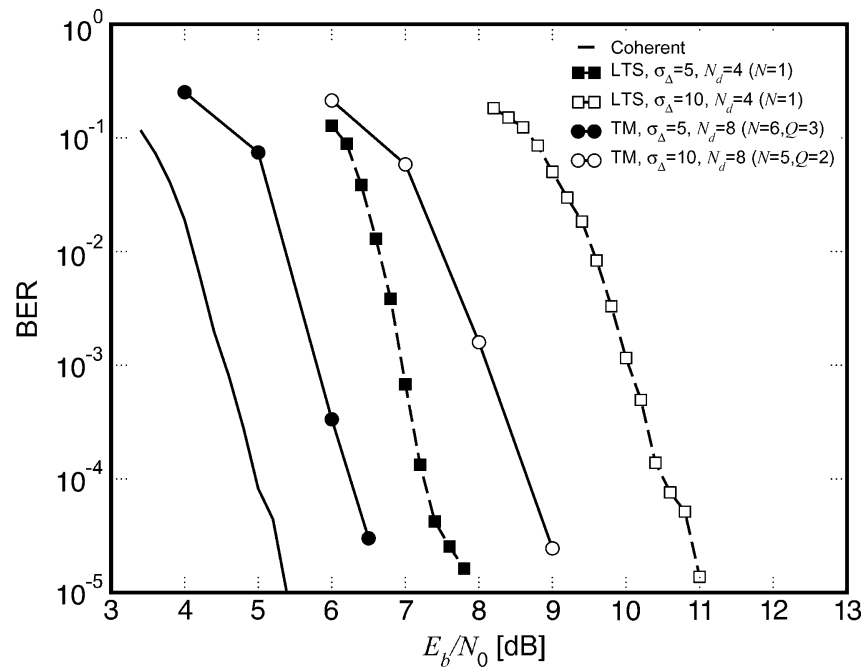

Fig. 5. BER of an SCCC with TM and LTS inner decoding algorithm, for phase-jitter standard-deviation $\sigma_{\Delta}=5^{\circ}$ and $\sigma_{\Delta}=10^{\circ}$. The spectral efficiency is $2 \mathrm{~b} / \mathrm{s} / \mathrm{Hz}$. For comparison, the performance of the equivalent coherent scheme is shown. In all cases, ten decoding iterations are considered.

length-1024 symbol interleaver [24]. The outer encoder is obtained by parallelizing two identical encoders with generator $G_{o}(D)$ as in (25) and puncturing every other coded bit. The inner code is essentially the antirotational invariant version of Code 1 in [25]. As in the previously considered SCCC, the three output bits are mapped to an 8-PSK symbol with natural mapping. The spectral efficiency of the overall code is then $2 \mathrm{~b} / \mathrm{s} / \mathrm{Hz}$. The performance of the LTS and TM schemes is shown in Fig. 5 and is compared with the performance of the equivalent coherent system. In all cases, ten decoding iterations are considered. In the LTS case, the best performance is obtained with $N_{d}=4$, while in the TM case, the best performance is obtained with $N_{d}=8$. In the latter case, for increasing phase dynamics the best performance is obtained by considering a reduced observation window $(N=5$ for $\sigma_{\Delta}=5^{\circ}$ and $N=4$ for $\sigma_{\Delta}=10^{\circ}$ ). Results on the FER (not shown here) revealed similar behavior.

The performance of the two considered detection strategies for this communication system has been further analyzed by evaluating the SNR that is necessary to obtain a prescribed BER of $10^{-3}$, at ten decoding iterations, as a function of the jitter standard-deviation $\sigma_{\Delta}$. Results are presented in Fig. 6. Two curves for each of the two detection strategies are shown. In the LTS case, one curve corresponds to $N_{d}=4$, while the other curve is obtained by optimizing the insertion rate for each specific value of the phase-noise-jitter standard-deviation $\sigma_{\Delta}$. In the TM case, both curves correspond to the optimized value $N_{d}=8$ : one corresponds to the case with $(N, Q)=(6,3)$, while the other curve refers to the case with $(N, Q)=(5,2)$. As one can see, the LTS detection strategy is better than the TM detection strategy for low-phase jitter standard deviation, while it worsens for increasing standard deviation. On the other hand, the TM approach is more robust at high phase dynamics and the required SNR to attain the desired BER is almost constant for $\sigma_{\Delta} \leq 5^{\circ}$. Moreover, the optimized value $N_{d}$ in the TM case does not seem to depend on the phase-jitter standard-devi-

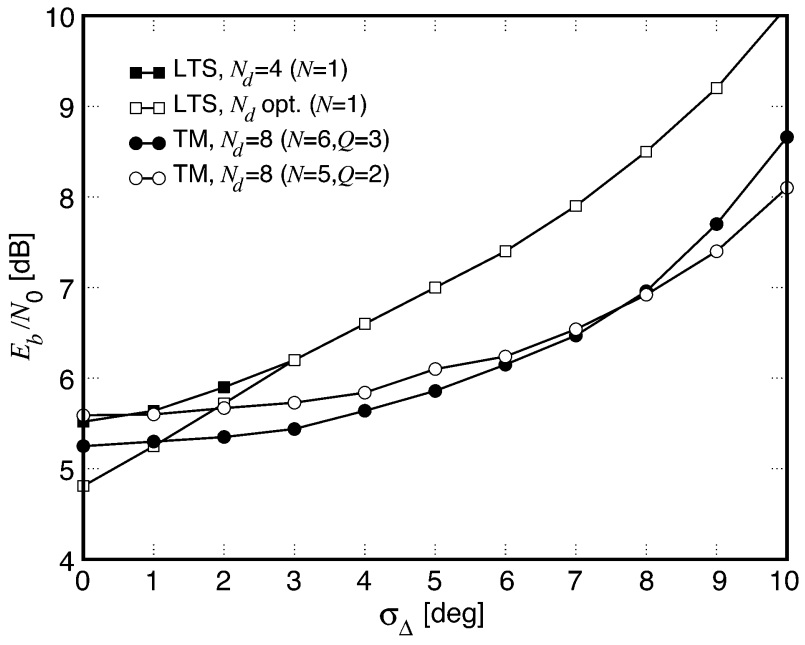

Fig. 6. $E_{b} / N_{0}$ required to obtain a BER of $10^{-3}$ at ten decoding iterations versus the phase-jitter standard-deviation $\sigma_{\Delta}$. Both the TM and LTS strategies are considered.



Fig. 7. BER of a PCCC with TM and LTS component decoding algorithms, for phase-jitter standard-deviation $\sigma_{\Delta}=5^{\circ}$ and $\sigma_{\Delta}=10^{\circ}$. For comparison, the performance of the equivalent coherent scheme is shown. In all cases, ten decoding iterations are considered. $N_{d}=16$ in all the adaptive cases.

ation $\sigma_{\Delta}$. As shown in Fig. 5, for increasing phase jitter, the TM scheme with a short observation window $(N=5)$ outperforms the one with a larger observation window $(N=6)$. The same analysis was carried out with the other considered communication systems, as well as with FER as the performance measure, and the same conclusion was reached: there is a threshold value for $\sigma_{\Delta}$ on the order of $1-2^{\circ}$, such that LTS is better than TM for $\sigma_{\Delta}$ values lower than the threshold and TM is better than LTS for $\sigma_{\Delta}$ values higher than the threshold.

In Fig. 7 the performance of the PCCC scheme with combined detection and decoding is shown. The component encoders are as in [1], with an inner pseudorandom interleaver of length 1024. BPSK modulation is used, resulting in an overall rate of $1 / 2$ and spectral efficiency of $0.5 \mathrm{~b} / \mathrm{s} / \mathrm{Hz}$. In all cases, 10 decoding iterations are considered. It can be seen that the performance of the LTS-based receiver is $1.5 \mathrm{~dB}$ worse than that of the TM-based receiver (at a BER of $10^{-3}$ ) for $\sigma_{\Delta}=5^{\circ}$, while the LTS-based receiver does not work at all for $\sigma_{\Delta}=10^{\circ}$. 


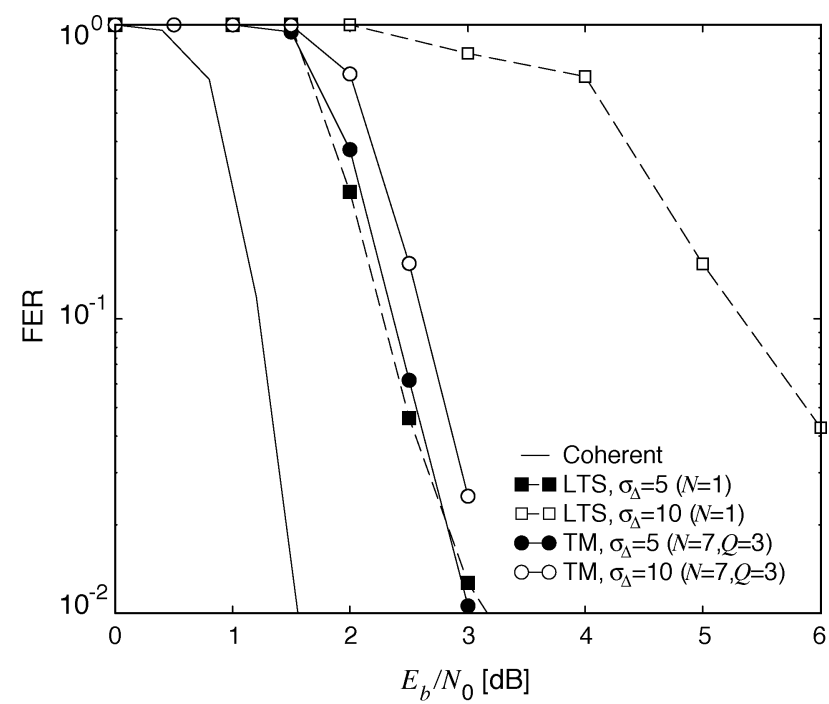

Fig. 8. FER of a PCCC with TM and LTS component decoding algorithm for phase-jitter standard-deviation $\sigma_{\Delta}=5^{\circ}$ and $\sigma_{\Delta}=10^{\circ}$. For comparison, the performance of the equivalent coherent scheme is shown. In all cases, ten decoding iterations are considered. $N_{d}=16$ in all the adaptive cases.

To further investigate this difference in performance between the TM and LTS schemes, the FER is shown in Fig. 8. The fact that the LTS scheme is slightly better than the TM scheme (for $\sigma_{\Delta}=5^{\circ}$ ) in terms of FER while the situation is reversed in terms of BER, shows that a catastrophic behavior is observed each time a frame error occurs in the LTS scheme, which is very likely related to the loss of lock in the PLL. This behavior was not noticed when considering SCCCs, in which case there is substantial agreement between the BER curves and the FER curves. A possible explanation is based on the fact that the first component decoder of the turbo receiver can use a reduced number of channel observables (due to puncturing). Hence, the very first iteration is less effective than in the SCCC case so that, if the observations are noisy, the LTS-based iterative receiver for a PCCC does not recover (in other words, the embedded PLL cannot recover the channel-phase rotation).

Finally, in Fig. 9, the performance of the PCCC-based separate detection and decoding scheme considered in Fig. 3 is shown. The purpose of these simulations is not to compare between separate and combined strategies (see [6] for such a comparison), but rather to compare LTS and TM strategies in the specific case of separate detection/decoding. In this case, the PCCC is almost identical to the combined scheme [1] described earlier. The only difference is that at every epoch the two output bits are mapped to a QPSK symbol with Gray mapping, resulting in an overall spectral efficiency of $1 \mathrm{~b} / \mathrm{s} / \mathrm{Hz}$. At the receiver side, the inner A-SODEM uses either the TM detection algorithm or the LTS detection algorithm. The iterative detection and decoding process can be characterized by $I_{e}$ external iterations between the A-SODEM and the inner turbo decoder and by $I_{i}$ internal iterations in the turbo decoder. The performance of the proposed adaptive algorithms is compared with the performance of the corresponding coherent scheme. In all cases, the number of external iterations $I_{e}$ is set to 5. In the LTS case, for $\sigma_{\Delta}=5^{\circ}$, the performance for $N=1$ (that is, there is only a single state) is shown, which is the same

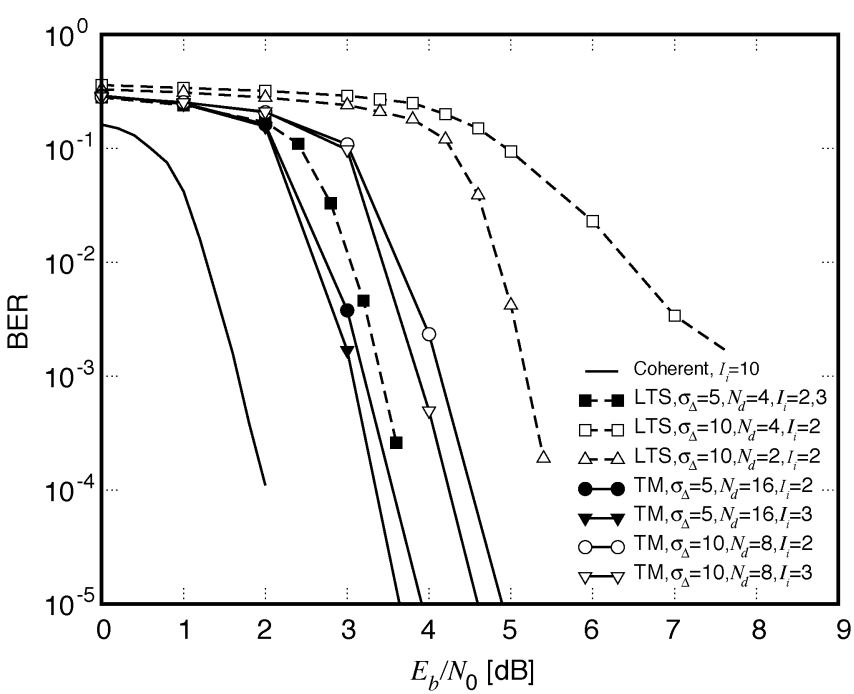

Fig. 9. BER of the separate scheme with rate-1/2 PCCC and QPSK output modulation. In all cases, $I_{e}=5$ external iterations between the A-SODEM and the turbo decoder are considered. Various numbers $I_{i}$ of inner decoding iterations are considered. In the LTS case, the adaptive algorithm is characterized by $N=1$ for $\sigma_{\Delta}={ }^{\circ}$ and by $N=2$ for $\sigma_{\Delta}=10^{\circ}$. In the TM case, the detection algorithm is characterized by $(N, Q)=(8,4)$.

for $I_{i}=2$ and $I_{i}=3$ internal decoding iterations. The best performance is obtained in this case by considering $N_{d}=4$. When increasing the phase jitter to $\sigma_{\Delta}=10^{\circ}$, the performance for $N=2$ and $I_{i}=2$ internal decoding iterations is shown. As one can see, the loss with respect to the coherent limit is significant. Increasing the pilot insertion rate to $N_{d}=2$, a performance improvement of approximately $3 \mathrm{~dB}$ is observed at a BER of $10^{-4}$. In the TM case, the A-SODEM uses the proposed noncoherent algorithm with $(N, Q)=(8,4): I_{i}=2$ and $I_{i}=3$ internal decoding iterations are considered. For $\sigma_{\Delta}=5^{\circ}$, the best performance is obtained with $N_{d}=16$, while for $\sigma_{\Delta}=10^{\circ}$ with $N_{d}=8$. Both for $\sigma_{\Delta}=5^{\circ}$ and $\sigma_{\Delta}=10^{\circ}$, increasing $I_{i}$ from 2 to 3 leads to a performance improvement of less than $0.3 \mathrm{~dB}$. The complexity with $\left(I_{e}, I_{i}\right)=(5,2)$ is roughly comparable to the performance of the perfect CSI scheme with $I_{i}=10$ decoding iterations. As one can see, the TM approach is more robust to strong phase variations with a reduced insertion rate with respect to the LTS case, i.e., with a reduced bandwidth expansion. However, this comes at the expense of an increased number of states in the A-SODEM ( $Q=4$ corresponds to 64 states).

\section{CONCLUSION}

In this paper, two different detection strategies were compared for the decoding of turbo codes in the presence of a random channel-phase rotation. The first is based on a limited-tree-search detection approach, while the second is based on a truncated-memory detection approach. In both cases, a suitable finite-state trellis can be identified. In the LTS adaptive algorithms, an explicit closed-loop phase estimate is considered. It is possible to interpret the TM adaptive algorithms as embedding an implicit open-loop phase estimate. The performance of the two detection algorithms was evaluated considering combined iterative detection and decoding schemes 
and separate detection and decoding schemes for SCCC and PCCC with various modulation formats. Extensive simulation results consistently showed that there is a threshold, in terms of the phase process standard deviation $\sigma_{\Delta}$, above (below) which the TM (LTS) detection strategy is more appropriate. The practical significance of this result is that, depending on the channel dynamics, the complexity, and the robustness requirements of a particular system, one should carefully choose one of the two strategies in order to optimize the performance/complexity tradeoff for the particular system under consideration. Moreover, in the case of the PCCC scheme, the two strategies compared differently in terms of BER and FER performance. This might suggest that the choice of the detection strategy should depend, in some cases, not only on the phase noise level but also on the specific system's performance measure to be maximized.

\section{REFERENCES}

[1] C. Berrou and A. Glavieux, "Near optimum error correcting coding and decoding: Turbo-codes," IEEE Trans. Commun., vol. 44, pp. 1261-1271, Oct. 1996.

[2] S. Benedetto, D. Divsalar, C. Montorsi, and F. Pollara, "Serial concatenation of interleaved codes: Performance analysis, design, and iterative decoding," IEEE Trans. Inform. Theory, vol. 44, pp. 909-926, May 1998.

[3] — "Soft-input soft-output modules for the construction and distributed iterative decoding of code networks," Eur. Trans. Telecommun. vol. 9, pp. 155-172, Mar./Apr. 1998.

[4] G. Ungerboeck, "Channel coding with multilevel/phase signals," IEEE Trans. Inform. Theory, vol. IT-28, pp. 55-67, Jan. 1982.

[5] L. Lu and S. W. Wilson, "Synchronization of turbo coded modulation systems at low SNR," in Proc. IEEE Int. Conf. Commun. (ICC), June 1998 , pp. 428-432.

[6] A. Anastasopoulos and K. M. Chugg, "Adaptive iterative detection for phase tracking in turbo coded systems, "IEEE Trans. Commun., vol. 49, pp. 2135-2144, Dec. 2001

[7] M. C. Valenti and B. D. Woerner, "Iterative channel estimation and decoding of pilot symbol assisted turbo codes over flat-fading channels," IEEE J. Select. Areas Commun., vol. 19, pp. 1697-1705, Sept. 2001.

[8] G. Colavolpe, G. Ferrari, and R. Raheli, "Noncoherent iterative (turbo) detection," IEEE Trans. Commun., vol. 48, pp. 1488-1498, Sept. 2000.

[9] L. R. Bahl, J. Cocke, F. Jelinek, and J. Raviv, "Optimal decoding of linear codes for minimizing symbol error rate," IEEE Trans. Inform. Theory, vol. IT-20, pp. 284-287, Mar. 1974.

[10] M. Peleg, S. Shamai (Shitz), and S. Galán, "Iterative decoding for coded noncoherent MPSK communications over phase-noisy AWGN channel," Proc. Inst. Elect. Eng.-Commun., vol. 147, pp. 87-95, Apr. 2000.

[11] C. Komninakis and R. D. Wesel, "Joint iterative channel estimation and decoding in flat correlated Rayleigh fading," IEEE J. Select. Areas Commun., vol. 19, pp. 1706-1717, Sept. 2001.

[12] A. Anastasopoulos and K. M. Chugg, "Adaptive soft-input soft-output algorithms for iterative detection with parametric uncertainty," IEEE Trans. Commun., vol. 48, pp. 1638-1649, Oct. 2000

[13] I. D. Marsland and P. T. Mathiopoulos, "On the performance of iterative noncoherent detection of coded M-PSK signals," IEEE Trans. Commun. vol. 48, pp. 588-596, Apr. 2000.

[14] G. Ferrari, "On iterative detection for channels with memory," Ph.D dissertation, Univ. Parma, Parma, Italy, Nov. 2001.

[15] M. D. Trott, S. Benedetto, R. Garello, and M. Mondin, "Rotational invariance of trellis codes-Part I: Encoders and precoders," IEEE Trans. Inform. Theory, vol. 42, pp. 751-765, May 1996.

[16] D. Raphaeli, "Noncoherent coded modulation," IEEE Trans. Commun., vol. 44, pp. 172-183, Feb. 1996.

[17] R. Raheli, A. Polydoros, and C. Tzou, "Per-survivor processing: A general approach to MLSE in uncertain environments," IEEE Trans. Commun., vol. 43, pp. 354-364, Feb./Apr. 1995.

[18] G. Colavolpe and R. Rabeli, "Noncoherent sequence detection," IEEE Trans. Commun., vol. 47, pp. 1376-1385, Sept. 1999.

[19] D. Divsalar and M. Simon, "Multiple-symbol differential detection of MPSK,” IEEE Trans. Commun., vol. 38, pp. 300-1308, Mar. 1990.
[20] G. Colavolpe and R. Raheli, "Theoretical analysis and performance limits of noncoherent sequence detection of coded PSK," IEEE Trans. Inform. Theory, vol. 46, pp. 1483-1494, July 2000.

[21] G. Ferrari, G. Colavolpe, and R. Raheli, "Noncoherent iterative decoding of spectrally efficient coded modulations," Ann. Telecommun., vol. 56, pp. 409-421, July/Aug. 2001.

[22] G. Colavolpe, G. Ferrari, and R. Raheli, "Reduced-state BCJR-type algorithms," IEEE J. Select. Areas Commun., vol. 19, pp. 848-859, May 2001.

[23] P. Thiennviboon, G. Ferrari, and K. M. Chugg, "Generalized trellisbased reduced-state soft-input/soft-output algorithms," in Proc. IEEE Int. Conf. Commun. (ICC), New York, Apr. 2002, pp. 1667-1671.

[24] D. Divsalar, S. Dolinar, and F. Pollara, "Serial concatenation of trellis coded modulation with rate-I inner code," in Proc. IEEE Global Telecommun. Conf. (GLOBECOM), San Francisco, CA, Nov. 2000, pp. 777-782.

[25] R. Nuriyev and A. Anastasopoulos, "Design and robustness analysis of rotationally invariant SCTCM," in Proc. IEEE Int. Conf. Commun. (ICC), Helsinki, Finland, June 2001.



Gianluigi Ferrari (S'97-M'03) was born in Parma, Italy, in November 1974. He received the "Laurea" (five-year program; summa cum laude) and Ph.D. degrees in electrical engineering from the University of Parma, in October 1998 and January 2002, respectively.

From July 2000 to December 2001, he was a Visiting Scholar at the Communication Sciences Institute, University of Southern California, Los Angeles. Since 2002, he has been a Research Professor at the Department of Information Engineering, University of Parma. Between November 2002 and February 2003 and between July 2003 and December 2003, he visited the Electrical and Computer Engineering Department, Carnegie Mellon University, Pittsburgh, PA, as a Research Associate His research interests include digital communication systems design, adaptive signal processing (with particular emphasis on iterative detection techniques for channels with memory), information theory, and ad hoc wireless networking.



Achilleas Anastasopoulos (S'97-M'99) was born in Athens, Greece, in 1971. He received the Diploma in electrical engineering from the National Technical University of Athens, Athens, Greece, in 1993 and the M.S. and Ph.D. degrees in electrical engineering from the University of Southern California, Los Angeles, in 1994 and 1999, respectively.

$\mathrm{He}$ is currently an Assistant Professor at the Department of Electrical Engineering and Computer Science, University of Michigan, Ann Arbor. He is the coauthor of the book Iterative Detection: Adaptivity, Complexity Reduction, and Applications (Reading, MA: Kluwer, 2001). His research interests are in the general area of communication theory, with emphasis in joint parameter estimation and data detection, adaptive iterative algorithms, and coding for fading channels.

Dr. Anastasopoulos is the recipient of the "Myronis Fellowship" in 1996 from the University of California and of the NSF CAREER Award in 2004. He served as a Technical Program Committee Member for ICC 2003. He currently serves on the Editorial Board of the IEEE TRANSACTIONS ON COMMUNICATIONS.

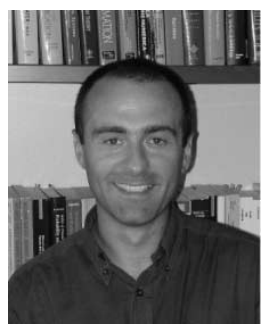

Giulio Colavolpe (S'96-A'00) was born in Cosenza, Italy, in 1969. He received the Dr. Ing. degree in telecommunications engineering (cum laude) from the University of Pisa, Pisa, Italy, in 1994 and the Ph.D. degree in information technology from the University of Parma, Parma, Italy, in 1998.

Since 1997, he has been with the University of Parma, where he is now an Associate Professor of Telecommunications. In 2000, he was Visiting Scientist at the Insitut Eurècom, Valbonne, France. His main research interests include digital transmission theory, channel coding, and signal processing. 


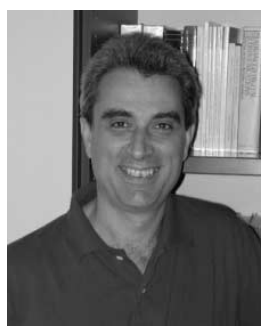

Riccardo Raheli (M'87) received the Dr. Ing. degree (Laurea) in electrical engineering (summa cum laude) from the University of Pisa, Italy, in 1983, the M.S. degree in electrical and computer engineering from the University of Massachusetts, Amherst, in 1986, and the Ph.D. degree (Perfezionamento) in electrical engineering (summa cum laude) from the Scuola Superiore di Studi Universitari e di Perfezionamento (now "S. Anna"), Pisa, in 1987.

From 1986 to 1988, he was a Project Engineer at Siemens Telecomunicazioni, Cassina de' Pecchi, Milan, Italy. From 1988 to 1991, he was a Research Professor at the Scuola Superiore di Studi Universitari e di Perfezionamento S. Anna, Pisa. In 1990, he was a Visiting Assistant Professor at the University of Southern California, Los Angeles. Since 1991, he has been with the University of Parma, Parma, Italy, first as a Research Professor, then as an Associate Professor, and currently as a Professor of Telecommunications His scientific interests are in the general area of statistical communication theory, with special attention to digital transmission systems, data-sequence detection techniques, digital signal processing, and adaptive algorithms for telecommunications. His research activity has lead to numerous scientific publications in leading international journals and conference proceedings, as well as a few industrial patents.

$\mathrm{He}$ has served on the Editorial Board of IEEE TRANSACTIONS ON COMMUNICATIONS as an Editor for Detection, Equalization, and Coding from 1999 to 2003. He is on the Editorial Board of the European Transactions on Telecommunications as an Editor for Communication Theory. 